\title{
Synthesis of Feedback Control Law for Stabilization of Chaotic System Oscillations by Means of Analytic Programming - Preliminary Study
}

\author{
${ }^{1}$ Roman Senkerik, ${ }^{1}$ Zuzana Oplatkova, ${ }^{2}$ Ivan Zelinka, ${ }^{2}$ Donald Davendra, ${ }^{1}$ Roman Jasek \\ ${ }^{I}$ Tomas Bata University in Zlin, Faculty of Applied Informatics, Nam T.G. Masaryka, Zlin, Czech Republic \\ ${ }^{2}$ Department of Computer Science, Faculty of Electrical Engineering and Computer Science, VB-TUO, 17, listopadu 15, \\ 70833 Ostrava-Poruba, Czech Republic \\ \{senkerik, oplatkova,zelinka, davendra, jasek\}@fai.utb.cz,ivan.zelinka@vsb.cz.
}

\begin{abstract}
This research deals with a synthesis of control law for selected discrete chaotic system - logistic equation by means of analytic programming. The novelty of the approach is that a tool for symbolic regression analytic programming - is used for the purpose of stabilization of higher periodic orbits - oscillations between several values of chaotic system. The paper consists of the descriptions of analytic programming as well as used chaotic system and detailed proposal of cost function used in optimization process. For experimentation, SelfOrganizing Migrating Algorithm (SOMA) with analytic programming and Differential evolution (DE) as second algorithm for meta-evolution were used.
\end{abstract}

Keywords: Chaos Control, Analytic programming, optimization, evolutionary algorithms. PACS: 02.60.Pn

\section{Introduction}

The interest about the interconnection between evolutionary techniques and control of chaotic systems is spread daily. First steps were done in [1] - [3] where the control law was based on Pyragas method: Extended delay feedback control - ETDAS [4]. These papers were concerned to tune several parameters inside the control technique for chaotic system. Compared to this, current presented research shows a possibility as to how to generate the whole control law (not only to optimize several parameters) for the purpose of stabilization of a chaotic system. The synthesis of control law is inspired by the Pyragas's delayed feedback control technique [5], [6]. Unlike the original OGY control method [7], it can be simply considered as a targeting and stabilizing algorithm together in one package [8]. Another big advantage of the Pyragas method for evolutionary computation is the amount of accessible control parameters, which can be easily tuned by means of evolutionary algorithms (EA). Instead of EA utilization [9], analytic programming (AP) is used in this research. AP is a superstructure of EAs and is used for synthesis of analytic solution according to the required behaviour. Control law from the proposed system can be viewed as a symbolic structure, which can be synthesized according to the requirements for the stabilization of the chaotic system. The advantage is that it is not necessary to have some "preliminary" control law and to estimate its parameters only. This system will generate the whole structure of the law even with suitable parameter values.

This work is focused on the expansion of AP application for synthesis of a whole control law instead of parameters tuning for existing and commonly used method control law to stabilize desired Unstable Periodic Orbits (UPO) of chaotic systems.

This research is an extension of previous research [10][12] focused on stabilization of simple p-1 orbit - stable state. In general, this research is concerned to stabilize p-2 UPO - higher periodic orbits (oscillations between two values).

Firstly, AP is explained, and then a problem design is proposed. The next sections are focused on the description of used cost function and evolutionary algorithms. Results and conclusion follow afterwards.

\section{Analytic Programming}

Basic principles of the AP were developed in 2001 [13]. Until that time only Genetic Programming (GP) and Grammatical Evolution (GE) had existed. GP uses Genetic Algorithms (GA) while AP can be used with any EA, independently on individual representation. To avoid any confusion, based on the nomenclature according to the used algorithm, the name - Analytic Programming was chosen, since AP represents synthesis of analytical solution by means of EA.

The core of AP is based on a special set of mathematical objects and operations. The set of mathematical objects is a set of functions, operators and so-called terminals (as well as in GP), which are usually constants or independent variables. This set of variables is usually mixed together and consists of functions with different number of arguments. Because of a variability of the content of this set, it is termed the "general functional set" - GFS. The structure of GFS is created by subsets of functions according to the number of their arguments. For example $\mathrm{GFS}_{\text {all }}$ is a set of all functions, operators and terminals, $\mathrm{GFS}_{3 \text { arg }}$ is a subset containing functions with

Proceedings of the Sixth Global Conference on Power Control and Optimization

AIP Conf. Proc. 1499, 196-202 (2012); doi: 10.1063/1.4768987

(C) 2012 American Institute of Physics 978-0-7354-1113-5/ $\$ 30.00$ 
only three arguments, $\mathrm{GFS}_{0 \text { arg }}$ represents only terminals, etc. The subset structure presence in GFS is vitally important for AP. It is used to avoid synthesis of pathological programs, i.e. programs containing functions without arguments, etc. The content of GFS is dependent only on the user. Various functions and terminals can be mixed together [13].

The second part of the AP core is a sequence of mathematical operations, which are used for the program synthesis. These operations are used to transform an individual of a population into a suitable program. Mathematically stated, it is a mapping from an individual domain into a program domain. This mapping consists of two main parts. The first part is called Discrete Set Handling (DSH) (Figure 1) [13] - [15] and the second one stands for security procedures which do not allow synthesizing pathological programs. The method of DSH, when used, allows handling arbitrary objects including nonnumeric objects like linguistic terms \{hot, cold, dark... \}, logic terms (True, False) or other user defined functions. In the AP, DSH is used to map an individual into GFS and together with security procedures creates the above-mentioned mapping, which transforms arbitrary individual into a program.

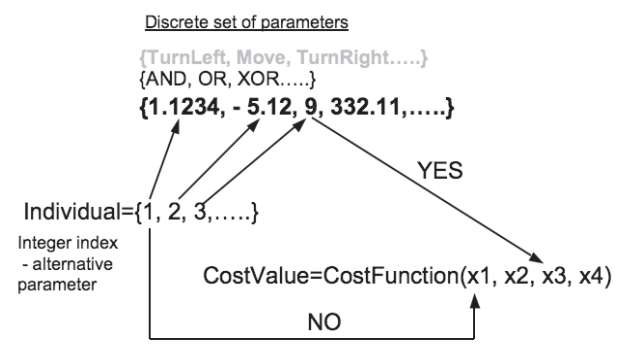

Figure 1 Discrete set handling

AP needs some EA [15] that consists of a population of individuals for its run. Individuals in the population consist of integer parameters, i.e. an individual is an integer index pointing into GFS. The creation of the program can be schematically observed in Figure 2. The individual contains numbers which are indices into GFS. The detailed description is represented in [13] - [15].

AP exists in 3 versions - basic without constant estimation, $\mathrm{AP}_{\mathrm{nf}}$ - estimation by means of nonlinear fitting package in Mathematica environment and $\mathrm{AP}_{\text {meta }}-$ constant estimation by means of another evolutionary algorithms; meta implies metaevolution.

\section{Problem design}

The brief description of used chaotic system and original feedback chaos control method ETDAS [4] is given here. The ETDAS control technique was used in this research as an inspiration for synthesizing a new feedback control law by means of evolutionary techniques.

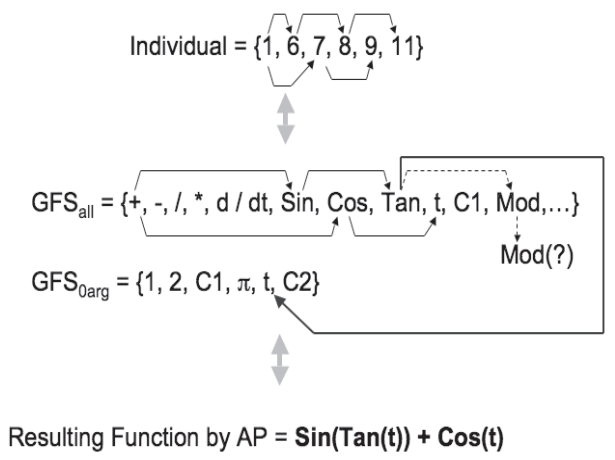

Figure 2 Main principles of AP

\subsection{Selected chaotic system}

The first of chosen examples of chaotic systems was the one-dimensional Logistic equation in form (1).

$x_{n+1}=r x_{n}\left(1-x_{n}\right)$

The Logistic equation (Logistic map) is a onedimensional discrete-time example of how complex chaotic behaviour can arise from very simple non-linear dynamical equation [16]. This chaotic system was introduced and popularized by the biologist Robert May [17]. It was originally introduced as a demographic model as a typical predator - prey relationship. The chaotic behaviour can be observed by varying the parameter $r$. At $r=3.57$ is the beginning of chaos. At $r>3.57$, the system exhibits chaotic behaviour. The example of this behaviour is depicted in bifurcation diagram - Figure 3.

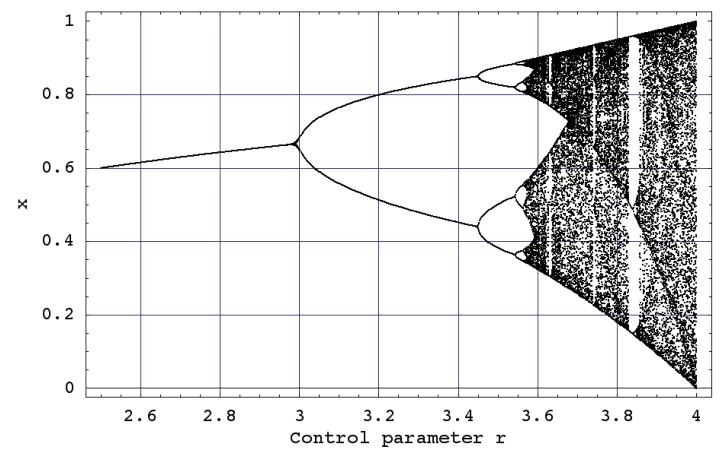

Figure 3. Bifurcation diagram of Logistic equation

\subsection{ETDAS control method}

This work is focused on explanation of application of AP for synthesis of a whole control law instead of demanding tuning of EDTAS [4] method control law to stabilize desired Unstable Periodic Orbits (UPO). In this research desired UPO is only p-2 (higher periodic orbit - oscillation between two values). ETDAS method was obviously an inspiration for preparation of sets of basic functions and operators for AP.

The original control method - ETDAS has form (2).

$F(t)=K\left[(1-R) S\left(t-\tau_{d}\right)-x(t)\right]$ 
$S(t)=x(t)+R S\left(t-\tau_{d}\right)$

Where: $K$ and $R$ are adjustable constants, $F$ is the perturbation; $S$ is given by a delay equation utilizing previous states of the system and $\tau_{d}$ is a time delay.

The original control method - ETDAS in the discrete form suitable for one-dimensional logistic equation has the form (3).

$$
\begin{aligned}
& x_{n+1}=r x_{n}\left(1-x_{n}\right)+F_{n} \\
& F_{n}=K\left[(1-R) S_{n-m}-x_{n}\right] \\
& S_{n}=x_{n}+R S_{n-m}
\end{aligned}
$$

Where: $m$ is the period of $m$-periodic orbit to be stabilized. The perturbation $F_{n}$ in equations (3) may have arbitrarily large value, which can cause diverging of the system outside the interval $\{0,1.0\}$. Therefore, $F_{n}$ should have a value between $-F_{\max }, F_{\max }$. In this preliminary study a suitable $F_{\max }$ value was taken from the previous research. To find the optimal value also for this parameter is in future plans.

Previous research concentrated on synthesis of control law only for $\mathrm{p}-1$ orbit (a fixed point). An inspiration for preparation of sets of basic functions and operators for AP was simpler TDAS [6] control method (4) and its discrete form suitable for logistic equation given in (5).

$$
\begin{aligned}
& F(t)=K[x(t-\tau)-x(t)] \\
& F_{n}=K\left(x_{n-m}-x_{n}\right)
\end{aligned}
$$

Compared to this work, the data set for AP presented in the previous research required only constants, operators like plus, minus, power and output values $x_{n}$ and $x_{n-1}$. Due to the recursive attributes of delay equation $S$ utilizing previous states of the system in discrete ETDAS (3), the data set for AP had to be expanded and cover longer system output history ( $x_{n}$ to $x_{n-9}$. ), thus to imitate inspiring control method for the successful synthesis of control law securing the stabilization of higher periodic orbits

\section{Cost Function}

Proposal for the cost function comes from the simplest Cost Function (CF) presented in [9]. The core of $\mathrm{CF}$ could be used only for the stabilization of $\mathrm{p}-1$ orbit. The idea was to minimize the area created by the difference between the required state and the real system output on the whole simulation interval $-\tau_{\mathrm{i}}$.

But another universal cost function had to be used for stabilizing of higher periodic orbit and having the possibility of adding penalization rules. It was synthesized from the simple $\mathrm{CF}$ and other terms were added. In this case, it is not possible to use the simple rule of minimizing the area created by the difference between the required and actual state on the whole simulation interval $-\tau_{\mathrm{i}}$, due to many serious reasons, for example: degrading of the possible best solution by phase shift of periodic orbit.
This CF is in general based on searching for desired stabilized periodic orbit and thereafter calculation of the difference between desired and found actual periodic orbit on the short time interval $-\tau_{\mathrm{s}}$ (approx. 20 - 50 iterations) from the point, where the first min. value of difference between desired and actual system output is found. Such a design of CF should secure the successful stabilization of either p-1 orbit (stable state) or higher periodic orbit anywise phase shifted.

The $\mathrm{CF}_{\text {Basic }}$ has the form (6).

$$
C F_{\text {Basic }}=\text { pen }_{1}+\sum_{t=\tau 1}^{\tau 2}\left|T S_{t}-A S_{t}\right|
$$

Where:

TS - target state

AS - actual state

$\tau_{1}$ - the first minimal value of difference between TS and AS

$\tau_{2}-$ the end of optimization interval $\left(\tau_{1}+\tau_{\mathrm{s}}\right)$

pen $_{1}=0$ if $\tau_{\mathrm{i}}-\tau_{2} \geq \tau_{\mathrm{s}}$;

pen $_{1}=10 *\left(\tau_{\mathrm{i}}-\tau_{2}\right)$ if $\tau_{\mathrm{i}}-\tau_{2}<\tau_{\mathrm{s}}$ (i.e. late stabilization)

\section{Used evolutionary algorithm}

This research used two evolutionary algorithms: Self-Organizing Migrating Algorithm [20] and Differential Evolution [21], [22]. Future simulations expect a usage of soft computing GAHC algorithm (modification of HC12) [18] and a CUDA implementation of $\mathrm{HC} 12$ algorithm [19].

\subsection{Self Organizing Migrating Algorithm - SOMA}

SOMA is a stochastic optimization algorithm that is modelled on the social behaviour of cooperating individuals [20]. It was chosen because it has been proven that the algorithm has the ability to converge towards the global optimum [20]. SOMA works with groups of individuals (population) whose behavior can be described as a competitive - cooperative strategy. The construction of a new population of individuals is not based on evolutionary principles (two parents produce offspring) but on the behavior of social group, e.g. a herd of animals looking for food. This algorithm can be classified as an algorithm of a social environment. To the same group of algorithms, Particle Swarm Optimization (PSO) algorithm can also be classified, sometimes called swarm intelligence. In the case of SOMA, there is no velocity vector as in PSO, only the position of individuals in the search space is changed during one generation, referred to as 'migration loop'.

The rules are as follows: In every migration loop the best individual is chosen, i.e. individual with the minimum cost value, which is called the Leader. An active individual from the population moves in the direction towards the Leader in the search space. At the end of the crossover, the position of the individual with minimum cost value is chosen. If the cost value of the new position is better than the cost value of an individual from the old population, the new one appears in new population. 
Otherwise the old one remains there. The main principle is depicted in Figures 4 - 6.

\subsection{Differential Evolution - DE}

DE is a population-based optimization method that works on real-number-coded individuals [21]. For each individual $\vec{x}_{i, G}$ in the current generation $\mathrm{G}, \mathrm{DE}$ generates a new trial individual $\vec{x}_{i, G}^{\prime}$ by adding the weighted difference between two randomly selected individuals $\vec{x}_{r 1, G}$ and $\vec{x}_{r 2, G}$ to a randomly selected third individual $\vec{x}_{r 3, G}$. The resulting individual $\vec{x}_{i, G}^{\prime}$ is crossedover with the original individual $\vec{x}_{i, G}$. The fitness of the resulting individual, referred to as a perturbed vector $\vec{u}_{i, G+1}$, is then compared with the fitness of $\vec{x}_{i, G}$. If the fitness of $\vec{u}_{i, G+1}$ is greater than the fitness of $\vec{x}_{i, G}$, then $\vec{x}_{i, G}$ is replaced with $\vec{u}_{i, G+1}$; otherwise, $\vec{x}_{i, G}$ remains in the population as $\vec{x}_{i, G+1}$. DE is quite robust, fast, and effective, with global optimization ability. It does not require the objective function to be differentiable, and it works well even with noisy and time-dependent objective functions. Description of used DERand1Bin strategy is presented in (7). Please refer to [21] and [22] for the description of all other strategies.

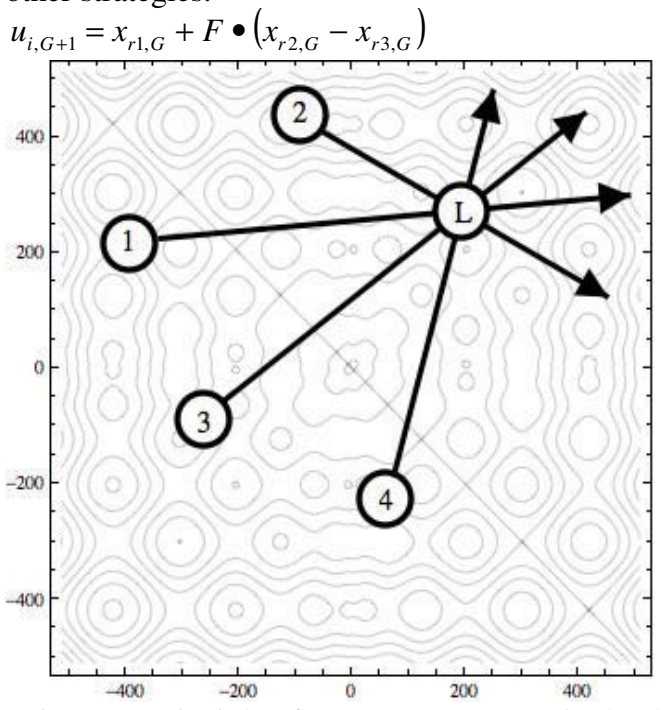

Figure 4. Principle of SOMA, movement in the direction towards the Leader

\section{Experimental results}

As described in section 2 about Analytic Programming, AP requires some EA for its run. In this paper $\mathrm{AP}_{\text {meta }}$ version was used. Meta-evolutionary approach means usage of one main evolutionary algorithm for AP process and second algorithm for coefficient estimation, thus to find optimal values of constants in the evolutionary synthesized control law.

SOMA algorithm was used for main AP process and DE was used in the second evolutionary process. Settings of EA parameters for both processes were based on performed numerous experiments with chaotic systems and simulations with $\mathrm{AP}_{\text {meta }}$ (Table 1 and Table 2).

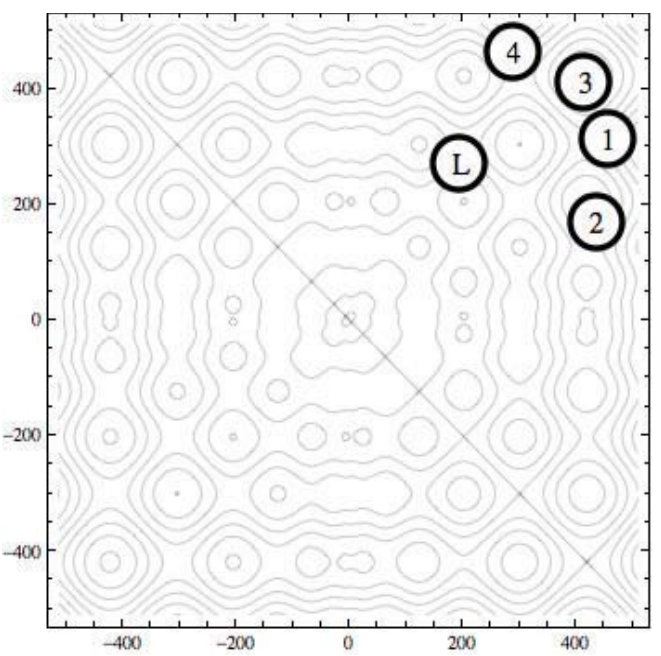

Figure 5. Principle of SOMA, the end of one migration loop

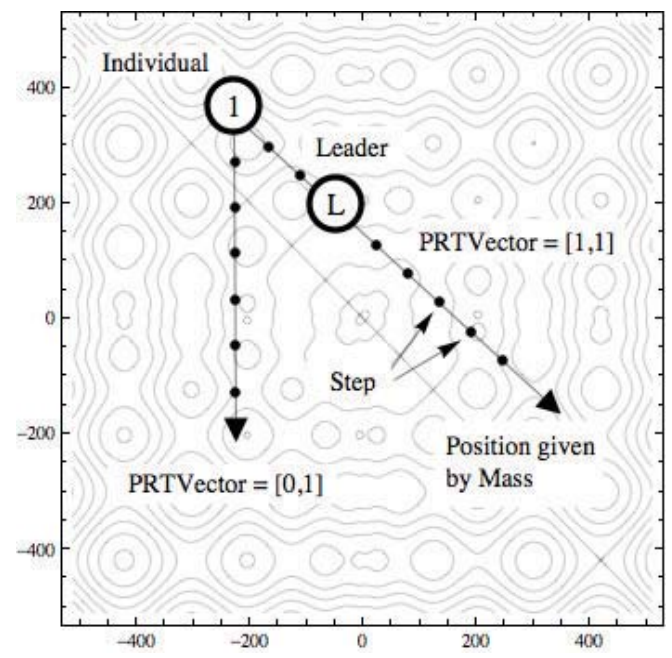

Figure 6. Basic principle of crossover in SOMA, PathLength is replaced here by Mass

Table 1. SOMA settings for AP

\begin{tabular}{|ll|}
\hline Parameter & Value \\
PathLength & 3 \\
Step & 0.11 \\
PRT & 0.1 \\
PopSize & 50 \\
Migrations & 4 \\
Max. CF Evaluations (CFE) & 5345 \\
\hline
\end{tabular}

Table 2. DE settings for meta-evolution

\begin{tabular}{|lc|}
\hline Parameter & Value \\
PopSize & 40 \\
F & 0.8 \\
CR & 0.8 \\
Generations & 150 \\
Max. CF Evaluations (CFE) & 6000 \\
\hline
\end{tabular}


The Analytic Programming used following setting-up:

Basic set of elementary functions for AP:

GFS2arg $=+,-, /, *, \wedge$

GFS0arg $=$ data $_{n-9}$ to data, $\mathrm{K}$

Within the research and this preliminary study a total number of 12 simulations was carried out. The most simulations were successful and have given new synthesized control law, which was able to stabilize the system at required behaviour ( $\mathrm{p}-2$ orbit) within short simulation interval of 200 iterations. Total number of cost function evaluations for AP was 5345, for the second EA it was 6000 , together 32.07 millions per each simulation. See Table 3 for simple CF values statistic.
Table 1: Cost Function values statistic

\begin{tabular}{|lc|}
\hline Statistical data & CF Value \\
Min & 0.0084 \\
Max & 0.5944 \\
Average & 0.3066 \\
Median & 0.3248 \\
\hline
\end{tabular}

The novelty of this approach represents the synthesis of feedback control law $F_{\mathrm{n}}(8)$ (perturbation) for the Logistic equation inspired by original ETDAS control method.

$x_{n+1}=r x_{n}\left(1-x_{n}\right)+F_{n}$

Table 4: Simulation results

\begin{tabular}{|cccccc|}
\hline Nr. & Control Law & Control Law with coefficients & CF Value & Figure \\
1 & $F_{n}=x_{n-6}-\frac{x_{n-6}}{K_{1}+x_{n-1}+x_{n}}$ & $F_{n}=x_{n-6}-\frac{x_{n-6}}{-0.264459+x_{n-1}+x_{n}}$ & 0.0084 & $7 \mathrm{a}$ \\
2 & $F_{n}=x_{n-6} x_{n}{ }^{\frac{K_{2} x_{n-5} x_{n-3}}{x_{n-8} x_{n-6} x_{n-1}}-K_{1} x_{n-5} x_{n-4}}$ & $F_{n}=x_{n-6} x_{n} \frac{62.739 x_{n-5} x_{n-3}-53.3298 x_{n-5} x_{n-4}^{33.7148}}{x_{n-8} x_{n-6} x_{n-1}}$ & 0.1546 & $7 \mathrm{~b}$ \\
3 & $F_{n}=x_{n-8} x_{n}{ }^{-\frac{K_{1} x_{n-6}\left(-x_{n-3}-x_{n-2}\right)}{x_{n-4}}}$ & $F_{n}=x_{n-8} x_{n}^{-\frac{23.389 x_{n-6}\left(-x_{n-3}-x_{n-2}\right)}{x_{n-4}}}$ & 0.1809 & $7 \mathrm{c}$ \\
4 & $F_{n}=x_{n}{ }^{K_{1}}$ & $F_{n}=x_{n}{ }^{28.1027}$ & 0.3249 & $7 \mathrm{~d}$ \\
\hline
\end{tabular}
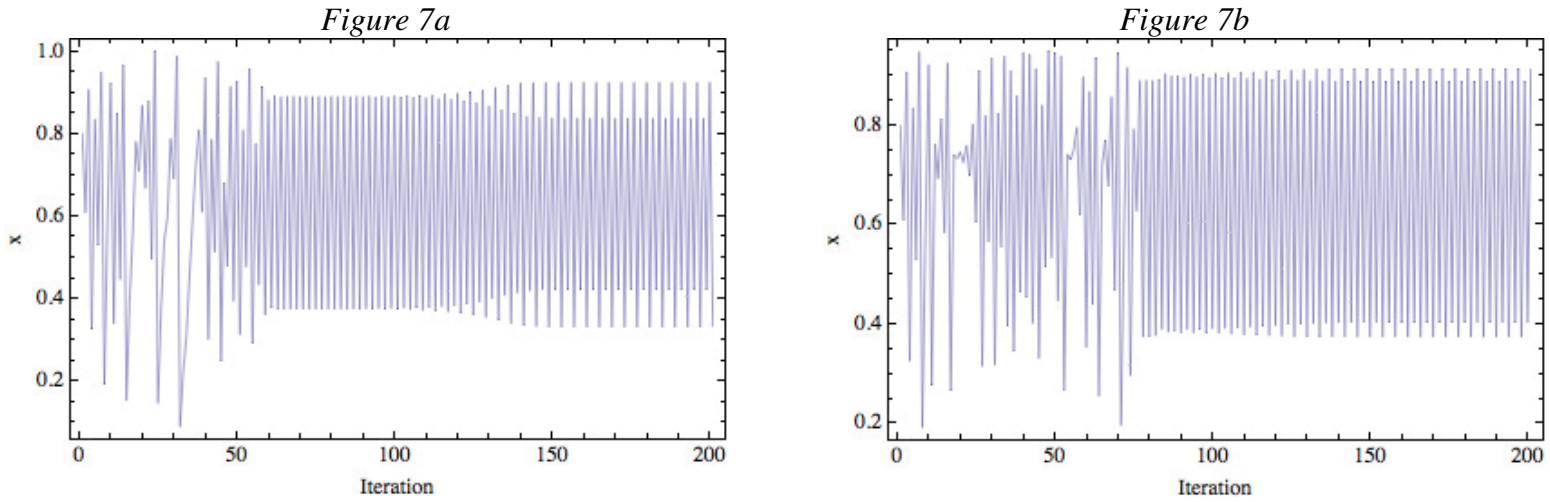

Figure $7 c$
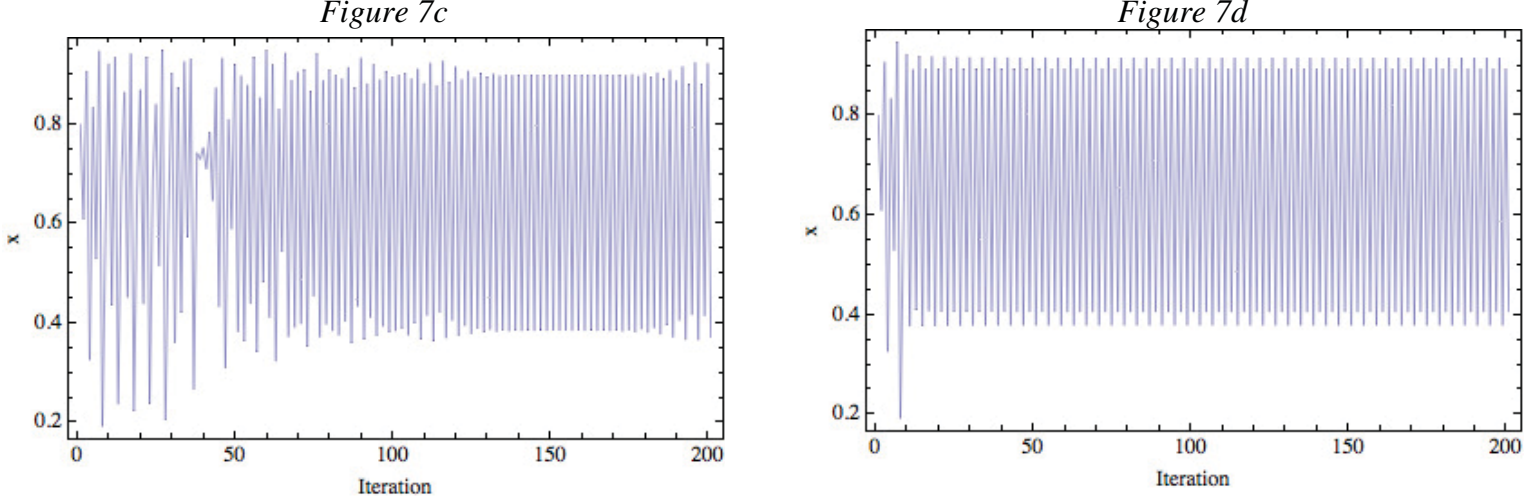

Figure 7: Examples of results - stabilization of chaotic system by means of control laws given in Table 4.

Following Table 4 contains four best examples of synthesized control laws. Since this is a preliminary study, obtained simulations results may evoke the impression, that the chaotic system was not fully stabilized on desired p-2 UPO (oscillations between 2 values). Due to the properties of unmodified cost function taken over from previous research, the system was stabilized only on short time interval and than escaped to either real or artificial 
controlled p-4 orbit. More about this phenomenon is written in conclusion section.

Based on the mathematical analysis, the real p-2 UPO for unperturbed logistic equation has following values: $x_{1}=0.3737, x_{2}=0.8894$.

Table 4 covers direct output from AP synthesized control law without coefficients estimated, further the notation with simplification after estimation by means of second algorithm DE, corresponding $\mathrm{CF}$ value, and identification of figure with simulation results.

\section{Conclusion}

This paper deals with a synthesis of a control law by means of AP for stabilization of selected chaotic system - Logistic equation at higher periodic orbit. In this presented approach, the analytic programming was used instead of tuning of parameters for existing control technique by means of EA's as in the previous research. Presented results reinforce the argument that AP is able to solve this kind of difficult problems and to produce a new synthesized control law in a symbolic way securing desired behaviour of chaotic system and stabilization.

An interesting phenomenon was discovered in simulation results. This work used the unmodified cost function taken over from previous research was in general based on searching for desired stabilized periodic orbit and thereafter calculation of the difference between desired and found actual periodic orbit on the short time interval. Therefore AP synthesized control laws, which followed this simple fact and secured the stabilization on part of this short time interval, and than the system freely escaped to either real or artificial controlled p-4 orbit. It is very interesting, that these control laws are able to stabilize the chaotic system on optional artificial periodic orbits. Most of common control method was developed for stabilization only on real UPO with low energy costs, thus, when the system enters the UPO, there is no perturbation. On the other hand, the above described phenomenon lends weight to the argument, that AP is a powerful symbolic regression tool, which is able to strictly and precisely follow the rules given by cost function and synthesize any symbolic formula, in the case of this research - the feedback controller for chaotic system. The question of energy costs and more precise stabilization will be included into future research together with development of better cost functions, different AP data set, and performing of numerous simulations to obtain more results and produce better statistics, thus to confirm the robustness of this approach.

\section{Acknowledgement}

This work was supported by the grant NO. MSM 7088352101 of the Ministry of Education of the Czech Republic and by grants of Grant Agency of Czech Republic GACR 102/09/1680 and by European Regional Development Fund under the project CEBIA-Tech No. CZ.1.05/2.1.00/03.0089.

\section{References}

[1] Zelinka I., Senkerik R., Navratil E., "Investigation on evolutionary optimization of chaos control", Chaos, Solitons \& Fractals, Volume 40, Issue 1, 2009, pp. 111-129.

[2] Senkerik R., Zelinka I., Navratil E., "Optimization of feedback control of chaos by evolutionary algorithms", in proc 1st IFAC Conference on analysis and control of chaotic systems, Reims, France, 2006, pp. 97 - 102 .

[3] Senkerik R., Zelinka I., Davendra D., Oplatkova Z., "Utilization of SOMA and differential evolution for robust stabilization of chaotic Logistic equation", Computers \& Mathematics with Applications, Volume 60, Issue 4, 2010, pp. 1026-1037.

[4] Pyragas K., "Control of chaos via extended delay feedback", Physics Letters A, vol. 206, 1995, pp. 323330.

[5] Just W., 1999, "Principles of Time Delayed Feedback Control", In: Schuster H.G., Handbook of Chaos Control, Wiley-Vch.

[6] Pyragas K., 1992, "Continuous control of chaos by self-controlling feedback", Physics Letters A, 170, pp. 421-428.

[7] Ott E., C. Greboki, J.A. Yorke, "Controlling Chaos", Phys. Rev. Lett. vol. 64, 1990, pp. 1196-1199.

[8] Kwon O. J., "Targeting and Stabilizing Chaotic Trajectories in the Standard Map", Physics Letters A. vol. 258, 1999, pp. 229-236.

[9] Senkerik, R., Zelinka, I., Oplatkova, Z.: Evolutionary Techniques for Deterministic Chaos Control, IEEE Computer Society, International Joint Conferences on computer, Information, and Systems Sciences, and Engineering, Bridgeport, Springer, 2010, pp. 391-396.

[10] Oplatkova, Z., Senkerik, R., Zelinka, I., Holoska, J.: Synthesis of Control Law for Chaotic Logistic Equation : Preliminary Study, IEEE, 4th International Conference on Mathematical Modelling and Computer Simulation, 2010,pp. 65-70.

[11] Oplatkova, Z., Senkerik, R., Zelinka, I., Holoska, J.: Synthesis of Control Law for Chaotic Henon System Preliminary Study, ECMS - Simulation Meets Global Challenges, Germany, 2010, pp. 277-282.

[12] Oplatkova, Z., Senkerik, R., Belaskova, S., Zelinka, I.: Synthesis of Control Rule for Synthesized Chaotic System by means of Evolutionary Techniques, 16th International Conference on Soft computing, Brno, 2010, pp. 91-98

[13]Zelinka I.,Oplatkova Z, Nolle L., Boolean Symmetry Function Synthesis by Means of Arbitrary Evolutionary Algorithms-Comparative Study, International Journal of Simulation Systems, Science and Technology, Volume 6, Number 9, August 2005, pp 44 - 56.

[14] Lampinen J., Zelinka I., New Ideas in Optimization Mechanical Engineering Design Optimization by Differential Devolution, Volume 1. London: McGraw-hill, 1999, 20 p.

[15] Oplatková, Z., Zelinka, I.: "Investigation on Evolutionary Synthesis of Movement Commands", 
Modelling and Simulation in Engineering, Volume 2009 (2009), 12 pages, Hindawi Publishing Corporation.

[16] Hilborn R.C., Chaos and Nonlinear Dynamics: An Introduction for Scientists and Engineers, Oxford University Press, 2000.

[17] May R.M., "Stability and Complexity in Model Ecosystems", Princeton University Press, 2001.

[18] Matousek R., „GAHC: Improved GA with HC station“, In WCECS 2007, San Francisco, pp. 915-920, 2007, ISBN: 978-988-98671-6-4.

[19] Matousek R, „HC12: The Principle of CUDA Implementation“. In MENDEL 2010, Mendel Journal series, pp. 303-308, 2010, ISBN: 978-80-214-41200. ISSN: 1803- 3814

[20]Zelinka I., "SOMA - Self Organizing Migrating Algorithm", In: New Optimization Techniques in Engineering, (B.V. Babu, G. Onwubolu (eds)), chapter 7, 33, Springer-Verlag, 2004

[21] Price, K. (1999), 'An Introduction to Differential Evolution', In: (D. Corne, M. Dorigo and F. Glover, eds.) New Ideas in Optimization, (pp. 79-108), London: McGraw-Hill.

[22] Price, K. and Storn, R. (2001), Differential evolution homepage,

[Online]: http://www.icsi.berkeley.edu/ storn/code.html, [Accessed 09/04/2011]. 
Copyright of AIP Conference Proceedings is the property of American Institute of Physics and its content may not be copied or emailed to multiple sites or posted to a listserv without the copyright holder's express written permission. However, users may print, download, or email articles for individual use. 